\title{
Endoscopic sleeve gastroplasty with 1-year follow-up: factors predictive of success
}

Authors

Institutions
G. Lopez-Nava' ${ }^{1}$ M. Galvao ${ }^{2}$, I. Bautista-Castaño ${ }^{1}$, J. P. Fernandez-Corbelle ${ }^{1}$, M. Trell ${ }^{1}$

${ }^{1}$ Bariatric Endoscopy Unit, Madrid Sanchinarro University Hospital

${ }^{2}$ Gastro Obeso Center, Gastrointestinal Surgery, São Paulo, Brazil submitted

6. September 2015

accepted after revision

23. November 2015

\section{Bibliography}

DOI http://dx.doi.org/

10.1055/s-0041-110771

Published online: 15.1.2016

Endoscopy International Open

2016; 04: E222-E227

(c) Georg Thieme Verlag KG

Stuttgart - New York

E-ISSN 2196-9736

\section{Corresponding author}

\section{Gontrand Lopez-Nava, MD}

Bariatric Endoscopy Unit

Madrid Sanchinarro University

Hospital

C/ San Enrique de Osso, 397

28055 Madrid

Spain

Fax: +34-91-7500203

glopeznava@digestivolopeznava.com
License terms

() (1) $\odot \circledast$
Background and study aims: Bariatric endoscopy has emerged as an aid in the nonsurgical treatment of obesity. The objective of this study is to critically provide the results and follow-up of endoscopic sleeve gastroplasty 1 year after the procedure.

Patients and methods: Prospective single-center follow-up study of 25 patients ( 5 men, 20 women) who underwent flexible endoscopic suturing for endoluminal gastric volume reduction. A multidisciplinary team provided post-procedure care. Patient outcomes were recorded at 1 year after the procedure. Linear regression analysis was done to evaluate the variables associated with best results at 1 year of follow-up.

Results: Mean body mass index (BMI) was 38.5 \pm $4.6 \mathrm{~kg} / \mathrm{m}^{2}$ (range $30-47$ ) and mean age $44.5 \pm 8.2$

\section{Introduction}

$\nabla$

Bariatric endoscopy has emerged for nonsurgical treatment of obesity, providing a treatment option for weight loss and associated comorbidities [1].

Fogel in 2008 [2] and Brethauer in 2010 [3] showed the feasibility of endoscopic gastric volume reduction for management of obesity using a superficial endoscopic suturing device that mimicked vertical banded gastroplasty surgical anatomy. In 2013, Abu Dayyeh and colleagues demonstrated the feasibility of creating a full endoscopic sleeve gastroplasty (ESG) that reduces the entire stomach through creation of a small-diameter sleeve along the lesser curvature of the stomach [4]. Since then, clinical experiences with ESG have been published. Outcomes of ESG have been published at 6 months $[5,6]$ and there is interest in outcomes with a longer follow-up period.

In this paper, we provide 1-year outcomes in the first 25 patients reaching this milestone and have identified predictors of favorable weight loss re- years (range 29-60). At 1 year, 22 patients continued with the follow-up ( 2 dropped out at 6 months and 1 at 3 months). There were no major intra-procedural, early, or delayed adverse events. Mean BMI loss was $7.3 \pm 4.2 \mathrm{~kg} / \mathrm{m}^{2}$, and mean percentage of total body weight loss was $18.7 \pm 10.7$ at 1 year. In the linear regression analysis, adjusted by initial BMI, variables associated with \% TBWL involved the frequency of nutritional $(\beta=$ $0.563, P=0.014)$ and psychological contacts $(\beta=$ $0.727, P=0.025$ ). The number of nutritional and psychological contacts were predictive of good weight loss results.

Conclusions: Endoscopic sleeve gastroplasty is a feasible, reproducible, and effective procedure to treat obesity. Nutritional and psychological interaction are predictive of success.

sponse to aid patient management moving forward.

\section{Patients and methods \\ $\nabla$}

Patients

All patients had failed lifestyle modification efforts. All procedures were conducted in accordance with good clinical practice and within the guidelines of the Declaration of Helsinki (WMA, 2004) [7] for studies using human subjects. Written informed consent was obtained from all patients.

The study was registered with the institutional review board of the Madrid Sanchinarro University Hospital. The registration number of the study is 657-GHM.

\section{Clinical Trial registration of clinical trial}

The study was registered in ClinicalTrials.gov with identifier NCT02231970.

Data were collected prospectively for analysis. The specific indications for the procedure were based 
on obesity parameters (body mass index [BMI] $30-49 \mathrm{~kg} / \mathrm{m}^{2}$ ) with previous failed attempts with conventional treatment of obesity and the willingness and ability of patients to be treated by a multidisciplinary team for at least 1 year. The procedure was contraindicated in patients with prior gastric surgery, potentially bleeding lesions (e.g., ulcers and acute gastritis) and neoplastic findings. Individuals with psychiatric disorders (mental retardation, manic-depressive psychosis, severe depression, schizophrenia, and untreated eating behavior disorders) that interfere with their ability to actively engage with the post-procedural instruction and recommended lifestyle adjustments were excluded. Coagulopathy and psychiatric disorders were excluded by blood tests and interviews with a psychologist, respectively.

\section{Endoscopic sleeve gastroplasty procedure}

As we have described previously (5), we refer to the technique as endoscopic endoluminal greater curvature plication. The procedure was performed with the patient in the left lateral decubitus position and under general anesthesia with endotracheal intubation. Pre-procedure antibiotics were given (Cefotaxima $2 \mathrm{~g}$ intravenously).

Construction of the gastroplasty was dependent on a cap-based flexible endoscopic suturing system (OverStitch; Apollo Endosurgery, Inc., Austin, Texas, USA), which was mounted onto a double-channel endoscope (GIF-2T160; Olympus Medical Systems Corp., Tokyo, Japan)placed through an esophageal overtube (US Endoscopy, Mentor, Ohio, USA) with carbon dioxide gas insufflation.

The goal of this procedure was to reduce the gastric lumen into a tubular configuration, with the greater curvature modified by a line of sutured plications as previously reported.

To perform the gastroplasty we deploy interrupted sutures from distal to proximal body. Each suture consists of six bites along the anterior/greater curvature/posterior gastric wall before it is cinched. Because this is not a continuous staple line, but rather, an invagination of the greater curvature of the stomach, intraluminal gaps exist along the plication line. These gap are of no clinical consequences as far as trapping food and are analogous to gaps seen with surgical plications of the greater curvature for weight loss. Reinforcing stiches are usually placed in the upper body of the stomach. The suture pattern has evolved from a very few cases addressing the fundus to the majority in which we leave the fundus open, so the patient can have a pouch and some accommodation ability.

Oral contrast studies assessed the gastroplasty at 24 hours for sleeve configuration and for potential suture line leak. Bleeding complications were excluded by blood tests at 6 hours and at 24 hours post-procedure. Post-procedure care remained unchanged from our earlier experience and included hospitalized observation, fasting sips, a liquid diet at 8 hours post-procedure, analgesia, and 24-hour discharge.

Adverse post-procedure events or bleeding (excluded by blood tests at 6 hours and 24 hours), were recorded as in our initial reported experience.

\section{Follow-up multidisciplinary bariatric team}

Post-procedure care with a nutritionist and a psychologist weekly or biweekly was maintained. Patient communication included personal interviews (face-to-face), telephone interviews, e-mails, and text messages.

The nutritional intervention changed during the course of treatment. Initially, the focus was on a transitional diet post-interven- tion. After patients started on solid food, the focus was on following the prescribed hypocaloric diet and discussing healthy food choices and alternatives. Once the first phase was completed, nutritional support shifted to providing patients with a workable diet program that they could follow over the long term, which was personalized to their individual needs.

The psychologist coached patients to follow the recommended lifestyle modification program necessary to maintain their weight loss over the long term. Furthermore, patients were coached on how to interact with food cues and obesogenic environment stimuli. Finally, they were taught how to recognize emotional eating cues and deal with them.

Gastric cavity restriction facilitates caloric limitation. Dietitians and psychologist were in continuous contact to resolve problems and to design the best strategy for treatment of each individual patient. Exercise was recommended, taking into account each patient's limitations and as prescribed by an exercise physiologist.

\section{Oral contrast studies}

Oral contrast studies were scheduled to assess the gastroplasty voluntarily at $3,6,9$, or 12 months.

\section{Endoscopic follow-up}

Gastroscopy was planned voluntarily at 6 or 12 months.

The data set collected for each patient was as follows:

1. Nutrition

a) Patient contacts: The number of contacts was divided into tertiles to study its relationship with the weight parameters. The tertiles were as follows: low adherence $=\mathrm{T} 1$, medium adherence $=\mathrm{T} 2$, and high adherence $=\mathrm{T} 3$.

b) Weight parameters: Baseline and follow-up examinations included assessment of weight and height as before, with BMI calculated as weight in kilograms divided by the square of height in meters. Outcomes at 1, 3, 6, and 12 months were: (1) change in body weight (TBWL); (2) percentage loss of initial body weight (\%TBWL); (3) percentage of excess body weight loss (percentage of weight lost compared with excess weight, defined as current weight minus the weight corresponding to a BMI of $25 \mathrm{~kg} / \mathrm{m}^{2}$ ) (\%EWL), and (4) change in BMI.

c) Post-procedure adverse events: Nausea, constipation, abdominal pain, hematemesis, melena, fever, reflux.

\section{Psychology}

a) Patient contacts: The number of contacts (face to face \& telephone) was divided into tertiles to study its relationship with the weight parameters. The tertiles were as follows: low adherence $=\mathrm{T} 1$, , edium adherence $=\mathrm{T} 2$, and high adherence $=\mathrm{T} 3$.

b) Behavioral measures (baseline and 1-year): (1) disorganized meals (2) "five meals a day" compliance, ( 3 ) speed eating, (4) snaking, (5) binge eating, (6) physical activity (PA), and (7) sleep quality.

\section{Post-procedure program structure}

\section{$\nabla$}

The programmatic follow up mirrored that which was applied to the initial pilot patient group reported earlier.

\section{First 4 weeks}

A liquid diet was started the day before the procedure and continued for 2 weeks, followed by progression from hypocaloric li- 
Table 1 Changes in weight-related parameters following endoscopic endolumenal greater curvature plication for the treatment of obesity at 3 months, 6 months, and 1 year post-procedure.

\begin{tabular}{|c|c|c|c|c|}
\hline Variable & $\begin{array}{l}1 \text { month } \\
\text { mean +SD } \\
n=25\end{array}$ & $\begin{array}{l}3 \text { months } \\
\text { mean +SD } \\
n=25\end{array}$ & $\begin{array}{l}6 \text { months } \\
\text { mean +SD } \\
n=24\end{array}$ & $\begin{array}{l}12 \text { months } \\
\text { mean +SD } \\
n=22\end{array}$ \\
\hline BMI $\left(\mathrm{kg} / \mathrm{m}^{2}\right)$ loss & $2.8 \pm 0.8$ & $4.9 \pm 1.6$ & $6.9 \pm 2.9$ & $7.3 \pm 4.2$ \\
\hline Total weight loss (kg) & $7.9 \pm 2.7$ & $14.1 \pm 5.5$ & $19.6 \pm 9.1$ & $21.1 \pm 12.6$ \\
\hline Percentage of weight loss (\%) & $7.4 \pm 2.3$ & $12.9 \pm 4.3$ & $17.8 \pm 7.5$ & $18.7 \pm 10.7$ \\
\hline Percentage of excess weight loss (\%) & $24.0 \pm 11.8$ & $40.5 \pm 16.5$ & $53.9 \pm 24.8$ & $54.6 \pm 31.9$ \\
\hline
\end{tabular}

BMI, body mass index

quids to small semisolid meals over 4 weeks. Exercise initially consisted of walking, with a progressive increase in intensity that paralleled the diet progression. Weekly contacts were made to evaluate performance and provide solutions for problems related to compliance with lifestyle treatment that patients may have experienced.

\section{Months 2-12}

Individual taste preferences were taken into account in designing hypocaloric diets.

Aerobic exercise was adjusted to patient capability and involved walking, jogging, cycling, aerobics, or swimming for a minimum of 30 minutes, 3 times a week. Patients were advised to add physical activity to any daily routine, (e.g. walking instead of taking mechanized transport and climbing stairs rather than using the elevator).

\section{Statistical analysis}

Descriptive analyses of the variables were performed using the test of proportions for qualitative variables and measurements of central tendency (mean) and measures of dispersion (standard deviation: s.d.) for quantitative variables.

The association between changes in the initial and final values of weight parameters used the student $t$ test for related pairs.

For comparisons of continuous variables, the comparisons of absolute means between groups were calculated using the student $t$ test.

Multivariate means and the $95 \%$ confidence interval (CI) for anthropometric measures at baseline according to tertiles of number of nutritionist and phycologist contacts were calculated using generalized linear models. The means were adjusted for age, sex, and initial BMI.

Finally, univariate linear regression analyses were fit to assess the association between \%TBWL and number of nutritional controls, and between \%TBWL and number of psychological controls, both controlling for initial BMI.

All $P$ values presented were two-tailed, and statistical significance was defined a priori at $P=0.05$. Data analyses were performed using SPSS 19.0 (SPSS Inc., Chicago, Illinois, USA).

\section{Results}

$\nabla$

The treatment group consisted of 25 patients ( 5 men, 20 women). Three patients dropped out, one at 3 months and two at 6 months. The final sample consisted of 22 patients with completed follow-up at 12 months.
Among the 25 patients, mean BMI was $38.5 \pm 4.6 \mathrm{~kg} / \mathrm{m}^{2}$ (range $30-47$ ) and mean age $44.5 \pm 8.2$ years (range $29-60$ ). The mean procedure time was 80 minutes (range $50-120$ minutes).

All patients underwent successful gastroplasty. There were no major intra-procedural, early, or delayed adverse events. No bleeding complications were found. During this period, patients received analgesics and antiemetics on an as-needed basis. Postdischarge pain (2-4 days) and nausea (1 day) were experienced by $50 \%$ and $20 \%$ of the patients, respectively. Oral contrast studies to assess the gastroplasty at 24 hours showed no leaking contrast and intact reductions. All patients were discharged the day after the procedure.

\section{Weight change}

- Table 1 shows the results of the evolution of the weight parameters. The initial parameters and the values collected at the postprocedure time intervals differed significantly. The largest decreases were seen in the first month after the procedure, when patients were on no solid foods.

\section{Nutritionist Follow-up}

During the first year of follow-up, the mean number of nutritional contacts was $19.6+9.9$ (range $3-32$ ). 0 Fig. 1 shows the weight loss parameters (BMI changes, \% TBWL, and \%EWL) across the tertiles of nutritional contacts. Tertiles are distributed as follows: T1 (3-16 contacts/year), T2 (17-24 contacts/year), and T3 (24-32 contacts/year).

As shown in $\bullet$ Fig. 1, the magnitude of the weight loss increased significantly $(P<0.05)$ in individuals who had more nutritional contacts. After adjustment for age, sex, and initial BMI, a linear trend was found for changes in \%TBWL $(P=0.045)$ and $\% \operatorname{EWL}(P=$ $0.013)$.

\section{Psychological follow-up}

During the first year of follow-up, the mean number of psychological contacts was $9.2+7.2$ (range $0-23$ ). Tertiles are distributed as follows: T1 ( $0-3$ contacts/year), T2 ( $4-12$ contacts/year), and T3 (13-23 contacts/year).

As shown in $\bullet$ Fig. 2, the magnitude of the weight loss increased significantly $(P<0.05)$ in individuals who had more psychological contacts. After adjustment for age, sex, and initial BMI, no linear trend was found for changes in \% TBWL and \%EWL.

\section{Changes in nutritional habits}

- Fig. 3 shows initial and final values for nutritional habits. Initially, the worse habits were "not eating 5 meals a day" $(94.1 \%)$ and "not eating slowly" (93.3\%). One year after the procedure, the most notable changes were "not eating 5 meals a day" (from $94.1 \%$ to $29.4 \%$ ) and binge eating (from $68.8 \%$ to $12.5 \%$ ). 


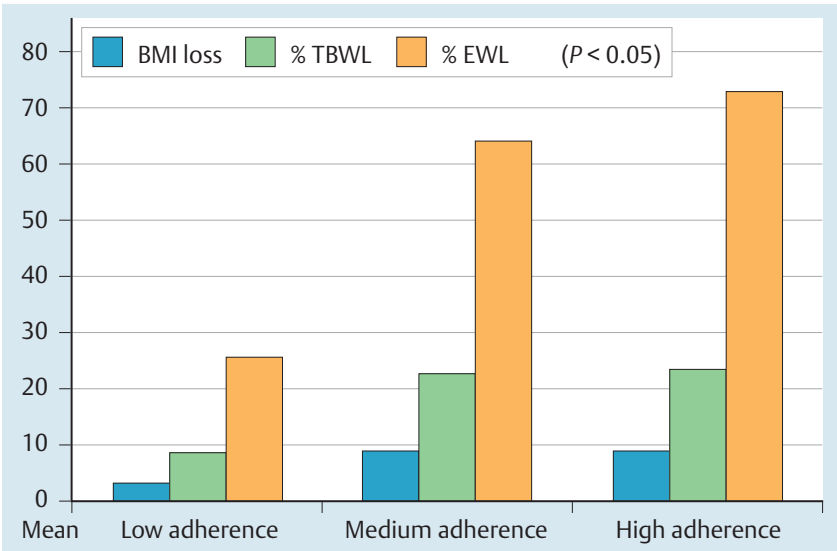

Fig. 1 Relationship between nutritional follow-up controls per year and weight loss parameters.

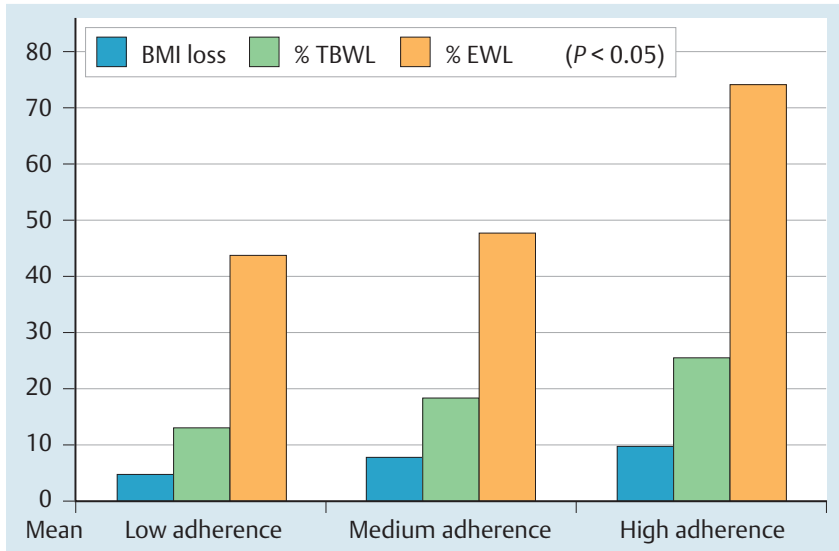

Fig. 2 Relationship between psychological follow-up controls per year and weight loss parameters.

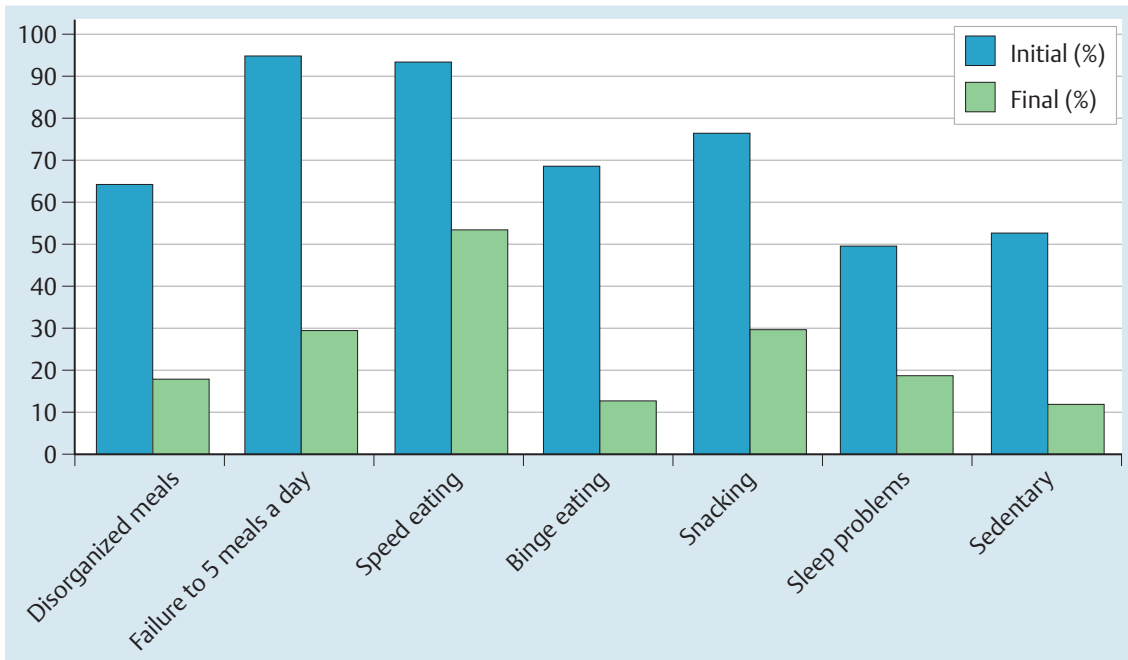

Fig. 3 Change in eating, sleep and sedentary habits: initial and 1-year prevalence.

Among the initially sedentary patients, $55.6 \%$ began physical activity (walking or doing cardiovascular exercises in the gym), and $75 \%$ of those who were initially not sedentary improved their level of physical activity (increasing walking time or doing other activities in the gym).

\section{Factors predictive of success}

The result of the linear regression analysis, controlling for initial BMI, showed that the number of nutritional contacts predict the \%TBWL (Beta $=0.563, P=0.014$ ) and that the number of psychological contacts predict the \%TBWL (Beta $=0.727, P=0.025$ ).

\section{Gastroplasty at 1 year}

- Fig. 4 shows the radiologic images at 24 hours and 1 year postprocedure. $\bullet$ Fig. 5 shows the endoscopy image at 1 year postprocedure.

Gastroplasty assessment was more successful than our earlier pilot experience and obtained in $90 \%$ of the patients, $50 \%$ endoscopically and $80 \%$ by contrast study. Based on these studies, one patient underwent a revision partial gastroplasty because of loosened plications. A tubular configuration of the gastroplasty was otherwise confirmed in the remaining patients.

\section{Discussion}

This extended experience demonstrates that endoscopic sleeve gastroplasty offers a safe and effective endolumenal weight loss option with durability at 1 year. The procedure does produce discomfort for patients in the immediate post-procedure period, with $50 \%$ experiencing moderate abdominal pain and $20 \%$ experiencing nausea, both of which can be controlled pharmacologically. No long-term complications were observed. At 1-year follow-up, patients reached $54.6 \%$ of EWL and $18.7 \%$ of TBWL. The subgroups with the highest number of nutritional and psychological interactions demonstrated the most favorable weight loss. This is not surprising, given our earlier pilot experience and general knowledge regarding the value of comprehensive supportive care post-procedure.

The study does have limitations. First, the sample size, although larger than the originally reported pilot group, is small. In addition, there is no control group in which the technique was not performed with which to compare results, although the patients who were treated persistently failed lifestyle modification. Regarding the demonstrated benefit of greater nutritional and psychological interaction, we are uncertain as to whether that was due to patient motivation stimulated by early post-procedure weight loss or if it is due to a unique motivational success of our nutritional and psychological programs. 


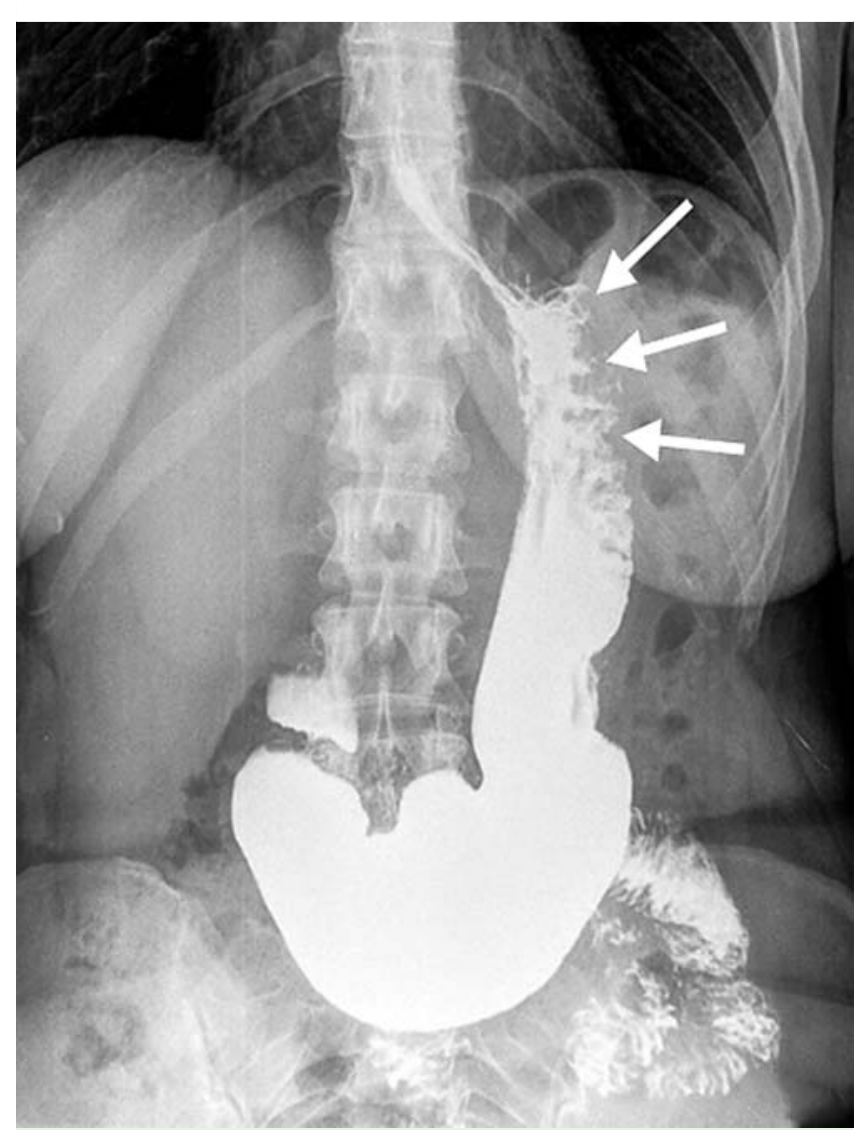

Fig. 4 Contrast Rx image of the endoscopic sleeve gastroplasty with the greater curvature modified by a line of sutured plications (the arrows indicate the location of the plications) at 1 year post-procedure

Determining how much of the contact needs to be face-face and how much electronic is a very important area of research. In our current limited sample size, we had to combine face-to-face and remote contacts to achieve adequate power to run the analysis. However, we have an active prospective protocol to answer this question.

Other bariatric endoscopic interventions are available, most notably, the intragastric balloon (IGB) [8-10]. The main difference between sleeve gastroplasty and IGB is treatment duration, with IGB a defined 6-month intervention. Retrospective studies with longer follow-up have shown maintenance of significant weight loss in only a quarter of the patients after balloon removal. These experiences suggest that long-term weight loss maintenance with IGB is difficult to achieve $[11,12]$.

The other technique for endoscopic gastric reduction is the Primary Obesity Surgery Endolumenal (POSE) procedure (USGI Medical, San Clemente, California, USA) [13,14]. The POSE method uses a per-oral Incisionless Operating Platform (IOP) for placement of multiple isolated transmural tissue anchor plications to reduce the gastric fundus and the gastric body. Results in 116 patients undergoing the POSE procedure with 1 year of follow-up [14] demonstrated a mean \%EWL of $44.9 \pm 24.4$. This procedure, however, does not provide an opportunity for reintervention as is the case with ESG

The durability of the Endoscopic Sleeve gastroplasty at 1-year, along with the weight loss results, suggest that this endolumenal technique remains effective and helpful. It should be noted that no irreversible anatomical alteration occurs in the gastric cavity and the technique is reproducible and repeatable. Therefore, re-

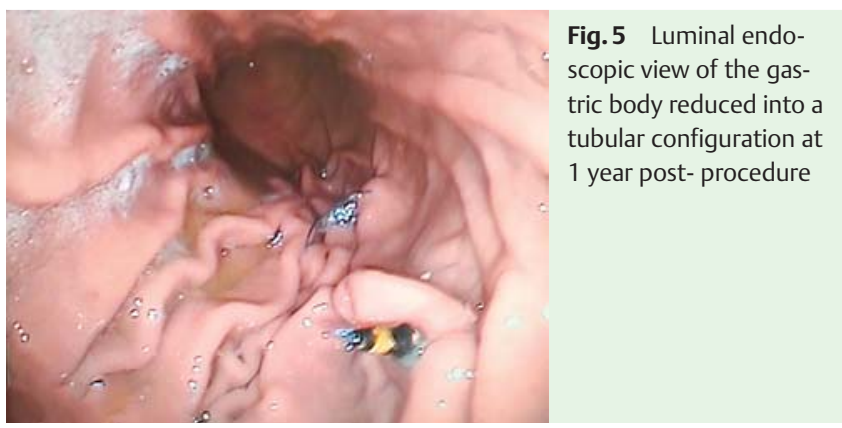

intervention in the future has the potential to achieve lasting results.

It is understood that dietary education, lifestyle modification, and physical exercise are essential in treatment of obesity. In 2year studies of dietary intervention for weight weight loss through different types of diets [15], the main predictor of weight loss was the number of checkups, regardless of other factors. Our experience confirms the findings from these prior studies. In our opinion, the current study serves to emphasize the importance of monitoring outcomes with these techniques and intervening when poor results in patients are associated with a low number of visits with the multidisciplinary team.

We can conclude that after 1 year, sleeve gastroplasty is an effective, safe, and well-tolerated procedure for treatment of patients with obesity, with regular monitoring by a multidisciplinary team a key measure to success.

Competing intersts: Dr. Lopez-Nava and Dr. Galvao are consultants for Apollo Endosurgery in the United States.

\section{References}

1 Abu Dayyeh BK, Thompson CC. Obesity and bariatrics for the endoscopist: New techniques. Therap Adv Gastroenterol 2011; 4: 433-442

2 Fogel R, De Fogel J, Bonilla Y et al. Clinical experience of transoral suturing for an endoluminal vertical gastroplasty: 1-year follow-up in 64 patients. Gastrointest Endosc 2008; 68: 51 - 58

3 Brethauer SA, Chand B, Schauer PR et al. Transoral gastric volume reduction for weight management: technique and feasibility in 18 patients. Surg Obes Relat Dis 2010; 6: 689-694

4 Abu Dayyeh BK, Rajan E, Gostout CJ. Endoscopic sleeve gastroplasty: a potential endoscopic alternative to surgical sleeve gastrectomy for treatment of obesity. Gastrointest Endosc 2013; 78: 530-535

5 Lopez-Nava G, Galvão MP, da Bautista-Castaño I et al. Endoscopic sleeve gastroplasty for the treatment of obesity. Endoscopy 2015; 47: 449452

6 Sharaiha RZ, Kedia P, Kumta $N$ et al. Initial experience with endoscopic sleeve gastroplasty: technical success and reproducibility in the bariatric population. Endoscopy 2015; 47: 164-166

7 WMA. (2004) World Medical Association Declaration of Helsinki, Tokyo.

8 Lopez-Nava G, Rubio MA, Prados $S$ et al. BioEnterics ${ }^{\circledR}$ intragastric balloon $(B I B \circledast)$. Single ambulatory center Spanish experience with 714 consecutive patients treated with one or two consecutive balloons. Obes Surg 2011; 21: 5-9

9 Machytka E, Klvana P, Kornbluth A et al. Adjustable intragastric balloons: a 12-month pilot trial in endoscopic weight loss management. Obes Surg 2011; 21: 1499-1507

10 Lopez-Nava G, Bautista-Castaño I, Jimenez-Baños A et al. Dual intragastric balloon: single ambulatory center Spanish experience with 60 patients in endoscopic weight loss management. Obes Surg 16. 05 2015: [Epub ahead of print] PubMed PMID: 25982804

11 Genco A, López-Nava G, Wahlen C et al. Multi-centre European experience with intragastric balloon in overweight populations: 13 years of experience. Obes Surg 2013; 23: 515-521 
12 Kotzampassi K, Grosomanidis V, Papakostas P et al. 500 intragastric balloons: What happens 5 years thereafter? Obes Surg 2012; 22: 896 903

13 Espinós JC, Turró R, Mata A et al. Early experience with the Incisionless Operating Platform (IOP) for the treatment of obesity: The Primary Obesity Surgery Endolumenal (POSE) procedure. Obes Surg 2013; 23: $1375-1383$
14 López-Nava Brevière G, Bautista-Castaño I, Jimenez A et al. The Primary Obesity Surgery Endolumenal (POSE) procedure: one-year patient weight loss and safety outcomes. Surg Obes Relat Dis 2015; 11: 861 865

15 Sacks FM, Bray GA, Carey VJ et al. Comparison of weight-loss diets with different compositions of fat, protein, and carbohydrates. N Engl J Med 2009; 360: $859-873$ 